INPLASY

PROTOCOL

To cite: Khairallah et al.

Delayed coloanal anastomosis technique in the management of low-lying rectal cancer: systematic review and metaanalysis. Inplasy protocol 202220002. doi: 10.37766/inplasy2022.2.0002

Received: 01 February 2022

Published: 01 February 2022

Corresponding author:

Sara Khairallah

sara.khairallah@ump.ac.ma

Author Affiliation:

Faculty of medecine and pharmacy, Mohamed I university, Oujda, Morocco.

Support: No financial support.

Review Stage at time of this submission: Completed but not published.

Conflicts of interest: None declared.

\section{Delayed coloanal anastomosis technique in the management of low- lying rectal cancer: systematic review and meta-analysis}

Khairallah, S1; Tijani, E².

Review question / Objective: Is there a difference in terms of post-operative events between delayed and immediate coloanal anastomoses in the management of rectum carcinoma?

Condition being studied: Rectal carcinoma.

Eligibility criteria: We defined the lower rectum as any rectal tumor located within $6 \mathrm{~cm}$ of the anal margin or within $2 \mathrm{~cm}$ of the upper edge of the sphincter ring.- All scientific articles published or not published between 01/1985 and 09/2021 that aim to demonstrate the postoperative, oncological and functional results of $A C A D$ in the curative treatment of adenocarcinoma of the lower rectum or rectal cancer including the lower rectum.- Scientific articles that discuss case series treated with ACAD in different benign or malignant pathologies, but where patient data and results of this procedure are well individualized in patients operated on rectal adenocarcinoma. - Abstracts of conference sessions, theses or unpublished articles (grey literature) with complete data, allowing their extraction and processing in our review.Translated with http://www.DeepL.com/Translator (free version).

INPLASY registration number: This protocol was registered with the International Platform of Registered Systematic Review and Meta-Analysis Protocols (INPLASY) on 01 February 2022 and was last updated on 01 February 2022 (registration number INPLASY202220002).

\section{INTRODUCTION}

Review question / Objective: Is there a difference in terms of post-operative events between delayed and immediate coloanal anastomoses in the management of rectum carcinoma?
Rationale: A number of observational and interventional studies in addition to 2 systematic reviews have recently demonstrated that delayed coloanal anastomosis is not inferior to immediate coloanal anastomosis with a diverting 
stoma. We conducted an updated metaanalysis of the current evidence on this topic.

Condition being studied: Rectal carcinoma.

\section{METHODS}

Search strategy: Study period: january 1985- december 2021; Language: French, English; Abstacting databases: PubMed, OVID, Scopus, LiSSa; Full-text databases: Cochrane central, clinicaltrials.gov Other search engines: google scholar.

Participant or population: Surgically treated with a urative intervention for low-lying rectal carcinoma.

Intervention: Delayed coloanal anastomosis.

Comparator: Immediate coloanal anastomosis with diverting stoma.

Study designs to be included: Observational and interventional studies.

Eligibility criteria: We defined the lower rectum as any rectal tumor located within $6 \mathrm{~cm}$ of the anal margin or within $2 \mathrm{~cm}$ of the upper edge of the sphincter ring.- All scientific articles published or not published between 01/1985 and 09/2021 that aim to demonstrate the postoperative, oncological and functional results of ACAD in the curative treatment of adenocarcinoma of the lower rectum or rectal cancer including the lower rectum.Scientific articles that discuss case series treated with ACAD in different benign or malignant pathologies, but where patient data and results of this procedure are well individualized in patients operated on rectal adenocarcinoma. - Abstracts of conference sessions, theses or unpublished articles (grey literature) with complete data, allowing their extraction and processing in our review.Translated with http:// www.DeepL.com/Translator (free version).

Information sources: PubMed, Scopus, OVID, PubMed, OVID, Scopus, LiSSa,
Cochrane central , clinicaltrials.gov, google scholar, cross referencing

Main outcome(s): Post-operative events(Mortality, Morbidity, ClavienDindo $>=3 b$, pelvic sepsis, anastomotic leak/fistula, colic ischemia, colic retraction, anastomotic stenosis, stoma confection) within 3 months after the first surgical intervention.

Quality assessment / Risk of bias analysis: Risk of bias tool(RoB 2) and MINORS score.

Strategy of data synthesis: PRISMA guidelines will be used for data reporting and risk of bias analysis by recommanded tools. Pooling odds ratios (OR) at $95 \%$ confidence intervals with heterogeneity verification and publication bias testing.

Subgroup analysis: Programmed but data were not available for analysis.

Sensitivity analysis: Programmed.

Language: English and French only.

Country(ies) involved: Morocco.

Keywords: Baulieux, technic, coloanal anastomosis, delayed, adenocarcinoma, neoplasm, cancer, low rectum, surgery, Turnbull Cutait.

Dissemination plans: Publication of the meta-analysis in a peer-reviewed journal and presentation in surgery conferences and meetings.

Contributions of each author:

Author 1 - Sara KHAIRALLAH Bibliographic search and date extraction and analysis. Scientific writing and publishing.

Email: sara.khairallah@ump.ac.ma

Author 2 - Tijani EL HARROUDI Supervision.

Email: el.harroudi@gmail.com 